\title{
Renal resistive index as a biomarker for acute kidney injury in aortic valve surgery
}

\author{
Thin Xuan Vo, Munir Boodhwani \\ Division of Cardiac Surgery, University of Ottawa Heart Institute, Ottawa, Canada \\ Correspondence to: Dr. Munir Boodhwani, MD, MMSc. Division of Cardiac Surgery, University of Ottawa Heart Institute, 40 Ruskin Street, Ottawa, \\ Ontario, Canada. Email: MBoodhwani@ottawaheart.ca. \\ Provenance: This is an invited Editorial commissioned by Executive Editor-in-Chief Jianxing He (Director of the Thoracic Surgery Department, The \\ First Affiliated Hospital of Guangzhou Medical University, Guangzhou, China). \\ Comment on: Andrew BY, Cherry AD, Hauck JN, et al. The Association of Aortic Valve Pathology With Renal Resistive Index as a Kidney Injury \\ Biomarker. Ann Thorac Surg 2018;106:107-14.
}

Submitted Aug 31, 2018. Accepted for publication Sep 11, 2018.

doi: $10.21037 /$ jtd.2018.09.45

View this article at: http://dx.doi.org/10.21037/jtd.2018.09.45

Acute kidney injury (AKI) is a common complication of cardiac surgery. Depending on the severity level, the incidence of AKI after cardiac surgery ranges from 3-30\%, with AKI requiring renal replacement therapy in the $3 \%$ range and mild AKI in the $30 \%$ range $(1,2)$. Several consensus guidelines and definitions have been developed to diagnose and grade AKI, including the Kidney Disease: Improving Global Outcomes (KDIGO) AKI guidelines, the Acute Kidney Injury Network (AKIN) guidelines, and the Risk-Injury-Failure-Loss-End-Stage Kidney Disease (RIFLE) criteria by the Acute Dialysis Quality Initiative Group (2-4). Several of these guidelines and definitions have been adopted and validated for the assessment, diagnosis, and reporting of AKI in several settings, including cardiac surgery. Based on these definitions, AKI of all severity levels, including mild AKI, are associated with increased short-term and long-term morbidity and mortality for patients undergoing cardiac surgery (1-4). Thus, it is imperative that the perioperative and surgical team ensure that the risk of AKI is minimalized during the perioperative period.

The pathophysiology of AKI in patients undergoing cardiac surgery is not completely understood. Several factors can contribute to the development of AKI in patients undergoing cardiac surgery including susceptibility to pre-renal azotemia, exposure to nephrotoxins such as intravenous contrast dye, decreased intraoperative renal perfusion, impairment of renal autoregulation, systemic inflammation related to cardiopulmonary bypass, hemodilution, microembolic events, and adrenergic/ neurohormonal activation (2). The multifactorial nature and limited understanding of the pathophysiology of AKI make it challenging to predict which patients are particularly susceptible. Serial measurements of serum creatinine and monitoring urine output are currently the most widely used methods of measuring and diagnosing AKI. The utility and validity of other biomarkers are unclear.

Various groups are currently investigating different approaches to predicting and detecting AKI in patients undergoing cardiac surgery. One approach is to measure a multitude of serum or urine biomarkers and correlate them with the development of AKI $(5,6)$. For example, Enger and colleagues found that increased baseline plasma levels of neopterin and $\mathrm{N}$-terminal pro-brain natriuretic peptide along with decreased levels of plasma lactoferrin were associated increased risk of developing AKI after cardiac surgery (5). However, a meta-analysis done by Ho et al. found that most serum and urine biomarkers were poor predictors of AKI in the perioperative period for cardiac surgery (6). The meta-analysis also revealed another important point-most current biomarkers are not measured intraoperatively, a period in which the surgical and perioperative team can intervene. Other approaches utilize patient demographic factors and comorbidities to predict the risk of developing severe AKI (7-9). For example, the widely used Cleveland Clinic 
Acute Renal Failure (ARF) score developed by Thakar et al. uses preoperative demographic and comorbidity data in addition to preoperative serum creatinine to predict the risk of developing AKI after cardiac surgery (9). Based on validation studies, these risk prediction models utilizing clinical data tend to be more predictive than biomarkers alone (6). An important limitation of these models, however, is that they are currently only predictive of severe AKI requiring renal replacement therapy. Though it is clear that a suitable predictor of AKI after cardiac surgery is needed, an ideal candidate has yet to be identified.

One potential biomarker that is currently being investigated for the prediction of AKI is the renal resistive index (RRI) via transabdominal or transesophageal ultrasonography. The RRI is a Doppler-derived value [RRI = (peak systolic velocity - end diastolic velocity)/peak systolic velocity] measured at the arcuate or interlobar arteries of the kidneys and provides an index of renal function. A threshold RRI of $>0.70$ is suggestive of decreased renal function $(10,11)$. Many studies have investigated the utility of RRI in the critically ill and perioperative population $(10,12)$. These studies are especially relevant in cardiac surgery patients given the higher rate of AKI in this patient population $(11,13,14)$. Benefits of RRI as a biomarker for the prediction of AKI include reproducibility, costeffectiveness, availability, and immediate feedback.

Andrew et al.'s retrospective study, published earlier this year in The Annals of Thoracic Surgery explores the nuances of aortic valve disease on the utility of the RRI in predicting the development of AKI post-cardiac surgery (15). Andrew et al. should be commended on their study, as they are the first group to report on the relationship between aortic valve pathology and RRI. In their study, patients were divided into four distinct groups depending on their predominant aortic valve pathology: normal aortic valve, predominantly aortic insufficiency (AI), predominantly aortic stenosis (AS), and combined insufficiency/stenosis (AI/AS). Determination of AKI was done using the KDIGO criteria. They find several relevant findings in their study population. Patients with predominantly $\mathrm{AI}$ or combined insufficiency/stenosis were found to have significantly elevated RRI pre-operatively. Yet, rates of AKI were not significantly different for the AI and AI/AS groups compared to the other groups.

Increased RRI found in patients with AI may be related to the hemodynamic disruption and decreased diastolic renal blood flow caused by aortic regurgitation. Similar to Andrew et al.'s findings, Sinning et al. also found elevated
RRI in their population of patients with moderate to severe paravalvular leak after transcatheter aortic valve intervention (TAVI) (16). However, the hemodynamic disruption and subsequent elevated RRI may not correlate clinically with increased rates of AKI as shown in Andrew et al.'s study. A clear explanation for this is not readily available at this time but the data does provide some further insight on the utility of RRI as a biomarker for AKI, especially in the setting of aortic valve pathology. As more studies investigate the utility of RRI as a biomarker for prediction of AKI, investigators must take into account the effects of regurgitant aortic lesions and adjust their RRI measurements as necessary. For example, Andrew et al. make the suggestion of decreasing the measured RRI depending on whether the patient has $\mathrm{AI}$ or AI/AS to more accurately predict the development of AKI, however validation of this remains to be completed. Perhaps, the use of RRI in the setting of lesions such as AI should be avoided altogether given the poor correlation of $\mathrm{RRI}$ and AKI in this population.

Several studies have already assessed the utility of RRI in the setting of cardiac surgery. A prospective cohort study done by Bossard et al. demonstrated that patients with increased RRI in the immediate post-operative period had an increased risk for the development of AKI (14). Increased RRI was also associated with worsening severity of AKI in their cohort. Interestingly, pre-operative RRI in this cohort was not associated with the development of AKI. Hertzberg et al. found that a pre-operative RRI of $>0.70$ was associated with a three-fold increase in risk of developing AKI after cardiac surgery (11). Regolisti et al. found a correlation between immediate post-operative RRI and the development of AKI after cardiac surgery. However, their receiver operating characteristic (ROC) analysis found that immediate post-operative RRI had poor predictive value for determining which patients would develop AKI (13). These studies in addition to Andrew et al.'s findings suggest that the RRI has great potential for prediction and earlier detection of AKI in patients undergoing cardiac surgery to allow for more expedient intervention. However, there are still issues to resolve prior to implementing RRI in routine clinical practice. Nonetheless, Andrew et al. provide further understanding on the consequences of AI on the measurement of RRI and help guide future studies on the utility of RRI as a predictor of AKI. Continued work to determine reliable predictors of AKI is essential for the guidance of therapies to prevent this common and potentially devastating complication of cardiac surgery (17-19). 


\section{Acknowledgements}

None.

\section{Footnote}

Conflicts of Interest: The authors have no conflicts of interest to declare.

\section{References}

1. Elmistekawy E, McDonald B, Hudson C, et al. Clinical impact of mild acute kidney injury after cardiac surgery. Ann Thorac Surg 2014;98:815-22.

2. Vives $M$, Wijeysundera $\mathrm{D}$, Marczin $\mathrm{N}$, et al. Cardiac surgery-associated acute kidney injury. Interact Cardiovasc Thorac Surg 2014;18:637-45.

3. Duthie FAI, McGeehan P, Hill S, et al. The utility of the additive EuroSCORE, RIFLE and AKIN staging scores in the prediction and diagnosis of acute kidney injury after cardiac surgery. Nephron Clin Pract 2014;128:29-38.

4. Petäjä L, Vaara S, Liuhanen S, et al. Acute Kidney Injury After Cardiac Surgery by Complete KDIGO Criteria Predicts Increased Mortality. J Cardiothorac Vasc Anesth 2017;31:827-36.

5. Enger TB, Pleym H, Stenseth R, et al. A Preoperative Multimarker Approach to Evaluate Acute Kidney Injury After Cardiac Surgery. J Cardiothorac Vasc Anesth 2017;31:837-46.

6. Ho J, Tangri N, Komenda P, et al. Urinary, plasma, and serum biomarkers' utility for predicting acute kidney injury associated with cardiac surgery in adults: A metaanalysis. Am J Kidney Dis 2015;66:993-1005.

7. Wijeysundera DN, Karkouti K, Dupuis JY, et al. Derivation and Validation of a Simplified. JAMA 2007;297:1801-9.

8. Mehta RH, Grab JD, O'Brien SM, et al. Bedside tool for predicting the risk of postoperative dialysis in patients undergoing cardiac surgery. Circulation 2006;114:2208-16.

9. Thakar CV, Arrigain S, Worley S, et al. A Clinical Score to Predict Acute Renal Failure after Cardiac Surgery. J Am Soc Nephrol 2005;16:162-8.

10. Schnell D, Darmon M. Renal Doppler to assess renal perfusion in the critically ill: A reappraisal. Intensive Care Med 2012;38:1751-60.
11. Hertzberg D, Ceder SL, Sartipy U, et al. Preoperative Renal Resistive Index Predicts Risk of Acute Kidney Injury in Patients Undergoing Cardiac Surgery. J Cardiothorac Vasc Anesth 2017;31:847-52.

12. Ninet S, Schnell D, Dewitte A, et al. Doppler-based renal resistive index for prediction of renal dysfunction reversibility: A systematic review and meta-analysis. J Crit Care 2015;30:629-35.

13. Regolisti G, Maggiore U, Cademartiri C, et al. Renal resistive index by transesophageal and transparietal echodoppler imaging for the prediction of acute kidney injury in patients undergoing major heart surgery. J Nephrol 2017;30:243-53.

14. Bossard G, Bourgoin P, Corbeau JJ, et al. Early detection of postoperative acute kidney injury by Doppler renal resistive index in cardiac surgery with cardiopulmonary bypass. Br J Anaesth 2011;107:891-8.

15. Andrew BY, Cherry AD, Hauck JN, et al. The Association of Aortic Valve Pathology With Renal Resistive Index as a Kidney Injury Biomarker. Ann Thorac Surg 2018;106:107-14.

16. Sinning JM, Adenauer V, Scheer AC, et al. Doppler-based renal resistance index for the detection of acute kidney injury and the non-invasive evaluation of paravalvular aortic regurgitation after transcatheter aortic valve implantation. EuroIntervention 2014;9:1309-16.

17. Meersch M, Schmidt C, Hoffmeier A, et al. Prevention of cardiac surgery-associated AKI by implementing the KDIGO guidelines in high risk patients identified by biomarkers: the PrevAKI randomized controlled trial (Intensive Care Med, 10.1007/s00134-016-4670-3). Intensive Care Med 2017;43:1749.

18. Liu Y, Sheng B, Wang S, et al. Dexmedetomidine prevents acute kidney injury after adult cardiac surgery: A metaanalysis of randomized controlled trials. BMC Anesthesiol 2018;18:7.

19. Chen X, Huang T, Cao X, et al. Comparative Efficacy of Drugs for Preventing Acute Kidney Injury after Cardiac Surgery: A Network Meta-Analysis. Am J Cardiovasc Drugs 2018;18:49-58.

Cite this article as: Vo TX, Boodhwani M. Renal resistive index as a biomarker for acute kidney injury in aortic valve surgery. J Thorac Dis 2018;10(Suppl 33):S4010-S4012. doi: 10.21037/jtd.2018.09.45 